\title{
Estimation of End Expiratory Volume using Stress Strain
}

\author{
A. Sundaresan ${ }^{1}$, C.E. Hann ${ }^{1}$, J.G. Chase ${ }^{1}$, G.M. Shaw ${ }^{2}$, \\ 1 Department of Mechanical Engineering, University of Canterbury, Christchurch, NZ \\ (e-mail:asu33@Canterbury.ac.nz). \\ 2 Department of Intensive Care, Christchurch Hospital, Christchurch, NZ
}

\begin{abstract}
Acute Respiratory Distress Syndrome (ARDS) results in collapse of alveolar units and loss of lung volume at end of expiration. Mechanical ventilation (MV) is used on patients with ARDS or Acute Lung Injury (ALI), with the end objective being to increase the end expiratory volume (EEV) and hence increasing overall functional residual capacity (FRC). Simple methods to estimate EEV at a given positive end expiratory pressure (PEEP) level in patients with ARDS/ ALI currently do not exist. Current viable methods are time consuming, relatively invasive and not model based. Previous studies have found a constant linear relationship between the global stress and strain in the lung independent of lung condition. This study utilizes the constant stress strain ratio and an individual patient's volume responsiveness to PEEP to estimate EEV. The estimation model identifies two global parameters that can be used to estimate EEV in a patient, $\beta$ and $\mathrm{m} \beta$. The parameter $\beta$ captures physiological parameters of FRC, lung and respiratory elastance, and varies depending on the PEEP level used, and $m \beta$ is the gradient of $\beta$ vs PEEP.
\end{abstract}

EEV was estimated at different PEEP values and compared to the measured EEV for 12 different patients with different levels of lung injury. The median percentage error is $18 \%$ (IQR: 6,49) for PEEP $=5 \mathrm{~cm}$ $\mathrm{H}_{2} \mathrm{O}, 10 \%$ (IQR: 9,18) for PEEP $=7 \mathrm{~cm} \mathrm{H}_{2} \mathrm{O}, 28 \%$ (IQR: 12,33) for PEEP $=10 \mathrm{~cm} \mathrm{H}_{2} \mathrm{O}, 3 \%$ (IQR: 2,10) for PEEP $=12 \mathrm{~cm} \mathrm{H}_{2} \mathrm{O}$ and $10 \%$ (IQR: 9,11) for PEEP $=15 \mathrm{~cm} \mathrm{H}_{2} \mathrm{O}$. The results were further validated using a cross correlation test and a linear regression was performed between the estimated and measured EEV with a median $\mathrm{R}^{2}$ of 0.948 (IQR: $0.915,0.968 ; 90 \%$ CI: $\left.0.814,0.984\right)$. This simple approach can be done at the bedside with minimal intervention to provide an estimate with reasonable accuracy to track patient condition.

\section{INTRODUCTION}

Acute lung injury (ALI) or its more severe form, acute respiratory distress syndrome (ARDS) (Hughes '00; Esteban '02; Walsh '04), have mortality rates from $20 \%$ to $70 \%$ (Bersten '02). ALI or ARDS occurs when the lung is inflamed and fills with fluid and cellular infiltrate causing a loss of functional lung units. This loss results in a stiffer and smaller lung - the so called "baby lung" (Gattinoni '05). Currently, there are no specific treatments for ALI/ ARDS. Management is supportive with an emphasis on minimising harm to the lungs from suboptimal mechanical ventilation (MV) (Ware '00).

MV is one of the main treatments for ARDS and is used to aid recovery by reducing or completely taking over the work of breathing. A positive end expiratory pressure (PEEP) is used to maintain a partially inflated lung at the end of expiration to maintain recruitment during subsequent breathing cycles (Amato '98; Network. '00; McCann '01; Halter '03). The optimal level of PEEP has been widely studied, but with no conclusive result (Amato '98).
Functional Residual Capacity (FRC) represents the inflated volume of the lung at end expiration at atmospheric pressure. In patients with ARDS, the objective of MV is to maximise the gas exchange and recruitment, while minimizing additional lung injury due to over distension. Thus a goal is to increase FRC through alveolar recruitment using PEEP.

The dynamic FRC is the new lung volume at atmospheric pressure, which incorporates this original FRC plus the extra recruitment due to MV with PEEP. Hence, this dynamic FRC value could be used as a clinical endpoint in ventilation management, with the potential to be continuously tracked with changes in patient condition.

Currently, there are few methods of measuring FRC at the bedside. Gas washout/ washin techniques are one method (Heinze '07), but are not readily available on most ventilators. A limitation of this measurement is that only an absolute value of FRC is measured and hence no information on the potential for new recruited lung volume is given. FRC can also be measured by using computed tomography (CT) scans (Malbouisson '01), but is not practical in the ICU. Thus, current methods estimating FRC are not standard ventilator functions, do not give the appropriate information required for optimal ventilator 
treatment, or require patient transport. Hence, there is no practical bedside method that can be used to estimate recruited lung. The ability to use standard ventilator data to estimate dynamic FRC, which includes recruited lung, would be a significant tool for managing MV. End expiratory volume (EEV) is the level of additional recruitment that is achieved in the lung due to an additional level of applied PEEP and is shown schematically in Figure 1. EEV also incorporates the level of FRC indirectly by giving an indication of lung recruitability

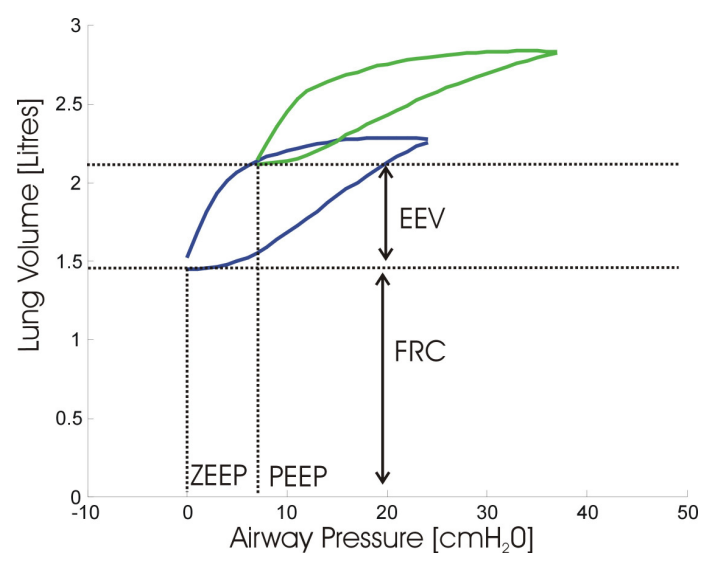

Figure 1 - Schematic showing the difference between FRC and EEV

This research develops a tool to estimate the level of additional recruitment of collapsed alveolar units resulting from changes made to the applied inspiratory and expiratory airway pressures during MV of an injured lung. The method is based on identifying global population parameters using a stress strain approach and estimating the level of additional volume recruitment due to PEEP. The potential for the model to be used in a clinical setting using retrospective clinical data is explored.

\section{METHODS}

Clinical data from 12 patients was obtained with different levels of lung injury or ARDS from Bersten et al (Bersten '98). These data include a minimum of 3 different PEEP values. Each set contains a measured EEV. The data was sampled for 60 seconds at $100 \mathrm{~Hz}$. After approximately 40 seconds of tidal ventilation at a given PEEP, the ventilator is then set to zero end expiratory pressure (ZEEP), allowing the lung to deflate to FRC. This manouver does not measure FRC as it includes the EEV of the extra recruitment due to PEEP.

Chiumello et al (Chiumello '08) studied the relationship between the global stress and strain during mechanical ventilation in ARDS patients. They defined the clinical equivalent of stress as the transpulmonary pressure $\left(\Delta P_{L}\right)$, while the strain was defined as the ratio of change in volume $(\Delta V)$ to the volume at the relaxed state of the lung or FRC. They also defined the specific lung elastance $\left(E_{\text {Lspec }}\right)$ as the transpulmonary pressure at which FRC effectively doubles.
The relationship between stress and strain is defined (Chiumello '08):

$$
\Delta P_{L}(\text { stress })=P_{L s p e c} \times \frac{\Delta V}{F R C}
$$

where $\triangle V / F R C$ is the strain and $E_{L S p e c}$ is the specific lung elastance. The values of the specific lung elastance $E_{L s p e c}$ in (Chiumello '08) are reported as $13.4 \pm 3.4$ for surgical control subjects, $12.6 \pm 3.0$ for medical control subjects, $14.4 \pm 3.6$ for ALI subgroup and $13.6 \pm 4.1 \mathrm{~cm} \mathrm{H}_{2} \mathrm{O}$ for the ARDS subgroup. This indicates that $E_{L s p e c}$ does not vary significantly within different groups and has a tight range of values.

The general relationship between the change in plateau airway pressure, $\Delta P_{a w}$, where the airflow is zero, and the corresponding transpulmonary pressure is (Chiumello '08):

$$
\begin{gathered}
\Delta P_{L}(\text { stress })=\Delta P_{a w} \times \alpha \\
\alpha=\frac{E_{L}}{E_{L}+E_{C W}}
\end{gathered}
$$

where $\alpha$ represents the static lung elastance, $E_{L}$ is the elastance of the lung, and $E_{C W}$ is the elastance of the chest wall. It is critical to understand the importance of $\alpha$ in $\mathrm{MV}$ therapy. When a given airway pressure is applied, part of the pressure is used to inflate the lungs, with the remaining used to inflate the chest wall. Although total elastance may be the same for two cases, if the lung elastance is higher, then a higher stress is undergone on the lung. Thus, $\alpha$ gives an indication of the severity of the ARDS affected lung.

The equation of motion is defined as:

$$
\Delta P_{a w_{-} t o t}=V \times E+Q \times R
$$

where $\Delta P_{a w_{-} \text {tot }}$ represents the total airway pressure, $V$ is the lung volume, $E$ is the respiratory elastance, $Q$ is the airflow rate and $R$ is the respiratory resistance (Carlo '99). The equation of motion describes the total airway pressure as a function of the resistive and elastic components of the respiratory system. In the case of no flow, the resistive term is zero and hence the pressure drop is shown in Equation (5).

$$
\Delta P_{a w}=V \times E
$$

At the beginning of inspiration, when the airway pressure is equal to PEEP, there is a point of zero flow, when the airflow reverses during expiration and inspiration. At this point in time, the volume is measured as EEV. Because plateau airway pressure occurs during zero flow, PEEP and the corresponding EEV could be used as a substitute for plateau pressure and volume.

Typically, if the pressure is measured, it is done at the ventilator or near the mask. Hence, all the PEEP data in this study represents plateau airway pressure. Thus, rather 
than using the transpulmonary pressure $\Delta \mathrm{P}_{\mathrm{L}}$, the airway pressure is used in this case.

Combining Equations (2) and (3) yields a formula for FRC involving the two easily measured quantities $\Delta \mathrm{V}$ and $\Delta \mathrm{P}_{\mathrm{aw}}$ $=\triangle P E E P$ assumed here:

$$
F R C=\frac{\Delta V}{\Delta P_{a w}} \times \frac{E_{L s p e c}}{\alpha}
$$

Equation (6) represents the FRC as a function of the specific lung elastance and the volume responsiveness of the patient. The data provided by Bersten et al did not include any FRC measurements, but included the extra volume recruited due to a given level of PEEP as a result of a recruitment manoeuvre or dynamic FRC. This is graphically represented in Figure 2, where FRC would be a value less than this EEV as shown schematically.

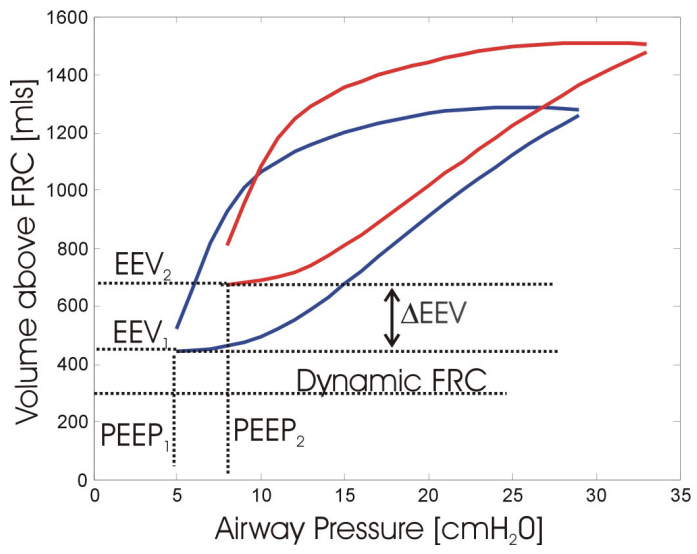

Figure 2 - Pressure Volume Curve showing the EEV relative to FRC

Figure 2 shows the EEV for a low and a high PEEP setting. The EEV is composed of FRC and the additional volume recruitment due to PEEP. As PEEP increases from $\mathrm{PEEP}_{1}$ to $\mathrm{PEEP}_{2}$, there is an increase in EEV which is represented by $\triangle \mathrm{EEV}$. Therefore physiologically, the $\triangle E E V$ represents the $\triangle F R C$ plus the change in alveolar recruitment due to PEEP. In this model, the FRC is not known, and the effect on FRC due to the recruitment manoeuvre is also not known.

The given PEEP and EEV in Equation (6) correspond to $\Delta \mathrm{V}=\Delta \mathrm{EEV}$ and $\Delta \mathrm{P}_{\mathrm{aw}}=\Delta P E E P$. Thus an alternative model for predicting EEV is:

$$
F R C+E E V=\frac{\Delta E E V}{\Delta P E E P} \times \frac{E_{L s p e c}}{\alpha}(1+x)
$$

Because $E_{L s p e c}$ and $\alpha$ are constant values, this suggests that these parameters can be lumped into a single parameter $\beta$ which is shown in Equation (8). Thus an alternative model to estimate $\mathrm{EEV}$ is:

$$
E E V=\frac{\Delta E E V}{\Delta P E E P} \times \beta
$$

Where:

$$
\beta=\frac{E_{L s p e c}}{\alpha} \times \frac{E E V}{F R C}
$$

And $\triangle E E V / \triangle P E E P$ indicates the volume responsiveness of the patient to the specified change in PEEP. Thus, for a given PEEP, $\beta$ can be assumed to be constant over all patients as $E_{\text {Lspec }}$ and $\alpha$ will remain constant for a given patient.

Because FRC was not known for any patient, $\beta$ was analytically solved based on Equation (8) using the measured EEV value from the data. Once $\beta$ values were calculated for each patient at each PEEP value, a median $\beta$ was then evaluated for each PEEP level. This median value was then approximated as a $\beta$ value for a given PEEP across the entire population. The EEV was then estimated using this median $\beta$ value. The process is summarised in Figure 3.

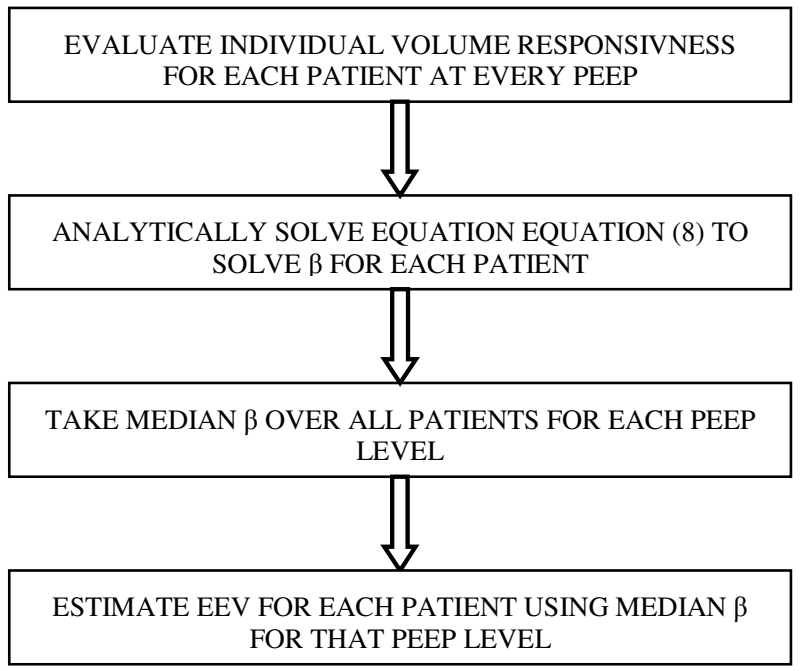

Figure 3 - Flow Chart showing the required process of EEV estimation

\section{RESULTS}

Table 1 shows the analytical solution of $\beta$ for each PEEP level over 12 patients. Due to the variability in $\beta$, a median value was obtained and is also shown along with the IQR. The EEV for each PEEP value was then estimated using this median value of $\beta$.

Table 2 shows the percentage error between the actual measured EEV and the EEV estimated with the model for all patients at all PEEP levels. Figure 4 shows a shows the general trend of clinical EEV vs predicted EEV for all the patients with a PEEP of $5 \mathrm{cmH}_{2} \mathrm{O}$. Using a linear regression, $\mathrm{R}^{2}=0.7187(\mathrm{R}=0.8478)$. This relatively low linear trend is attributed to the limited number of data points and limited clinical EEV range. 
Table 1 - Exact and Median Values of $\beta$ for different PEEP across all twelve patients

\begin{tabular}{|c|c|c|c|c|c|}
\hline PEEP [ $\left.\mathrm{cm} \mathrm{H} \mathrm{H}_{2} \mathrm{O}\right]$ & 5 & 7 & 10 & 12 & 15 \\
\hline & 4.67 & 7.14 & 10.48 & 11.79 & 15.17 \\
\hline & 5.31 & 7.96 & 11.09 & 12.23 & 12.43 \\
\hline & 6.10 & 5.25 & 8.00 & 8.98 & \\
\hline & 2.96 & 6.75 & 9.12 & 11.49 & \\
\hline & 4.96 & 3.58 & 6.79 & 10.28 & \\
\hline & 2.07 & 6.51 & 9.50 & 12.44 & \\
\hline & 4.49 & 5.67 & 10.13 & 11.94 & \\
\hline & 3.15 & 7.23 & 9.75 & & \\
\hline & 4.73 & 5.95 & 8.86 & & \\
\hline & 3.85 & & & & \\
\hline & 2.43 & & & & \\
\hline Median & 4.49 & 6.51 & 9.31 & 11.79 & 13.80 \\
\hline$I Q R$ & $\begin{array}{l}{[3.06,} \\
4.85]\end{array}$ & $\begin{array}{r}{[5.67,} \\
7.14]\end{array}$ & $\begin{array}{l}\text { [8.22, } \\
10.04]\end{array}$ & $\begin{array}{l}{[10.89,} \\
12.09]\end{array}$ & $\begin{array}{l}{[13.12,} \\
14.49]\end{array}$ \\
\hline
\end{tabular}

Table 2 - Absolute percent error of predicted EEV vs clinical EEV for all twelve patients at all PEEP levels

\begin{tabular}{|c|c|c|c|c|c|}
\hline PEEP [cm $\left.\mathbf{H}_{\mathbf{2}} \mathbf{O}\right]$ & $\mathbf{5}$ & $\mathbf{7}$ & $\mathbf{1 0}$ & $\mathbf{1 2}$ & $\mathbf{1 5}$ \\
\hline & 3 & 9 & 9 & 0 & 9 \\
\hline & 25 & 18 & 41 & 3 & 11 \\
\hline & 14 & 24 & 19 & 32 & \\
\hline & 54 & 4 & 29 & 3 & \\
\hline & 8 & 82 & 4 & 15 & \\
\hline & 119 & 0 & 32 & 5 & \\
\hline & 1 & 15 & 36 & 1 & \\
\hline & 45 & 10 & 33 & & \\
\hline & 4 & 9 & 27 & & \\
\hline & 18 & & & & \\
\hline & 87 & & & & \\
\hline & $\mathbf{1 8}$ & $\mathbf{1 0}$ & $\mathbf{2 8}$ & $\mathbf{3}$ & $\mathbf{1 0}$ \\
\hline & {$[\mathbf{6 , 4 9 ]}$} & {$[\mathbf{9 , 1 8}]$} & {$[\mathbf{1 2 , 3 3 ]}$} & {$[\mathbf{2 , 1 0 ]}$} & {$[\mathbf{9 , 1 1 ]}$} \\
\hline
\end{tabular}

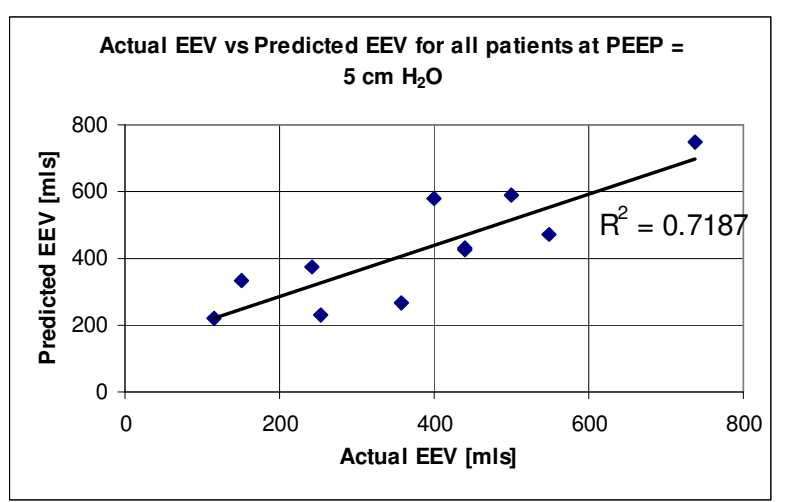

Figure 4 - Plot of Actual EEV vs Predicted EEV for all patients with a PEEP of $5 \mathrm{cmH}_{2} \mathrm{O}$
Rather than using just the EEV from a PEEP of 5, Figure 5 shows a higher level of linearity if predicted and actual EEV measurements from all patients at all PEEP values are used with an $\mathrm{R}^{2}=0.9466(\mathrm{R}=0.9729)$. The relatively lower $\mathrm{R}^{2}$ value in Figure 4 could also be attributed to the relatively low range of EEV values as compared Figure 5. In particular, Figure 4 shows an actual EEV ranging between 100 and $800 \mathrm{mls}$, Figure 5 shows an actual EEV range from 50 to $2000 \mathrm{mls}$.

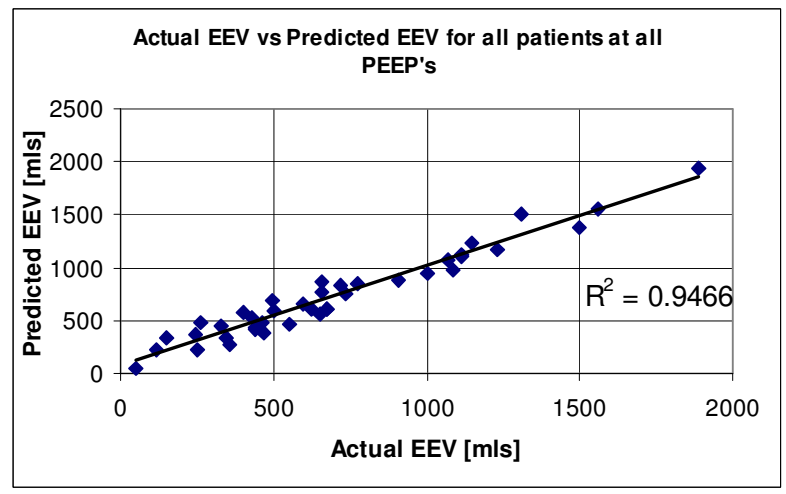

Figure 5 - Actual vs Predicted EEV for all patients and PEEP

\section{VALIDATION}

The model based method was validated using a correlation test with the measured EEV obtained for each patient. The data provided 40 different EEV measurements at various PEEP levels. Thus, $80 \%$ of the data (32 data points) were randomly selected without replacement and the median $\beta$ value found for each PEEP along with the gradient of $\beta$ vs PEEP, or $\mathrm{m} \beta$ as illustrated on Figure 6.

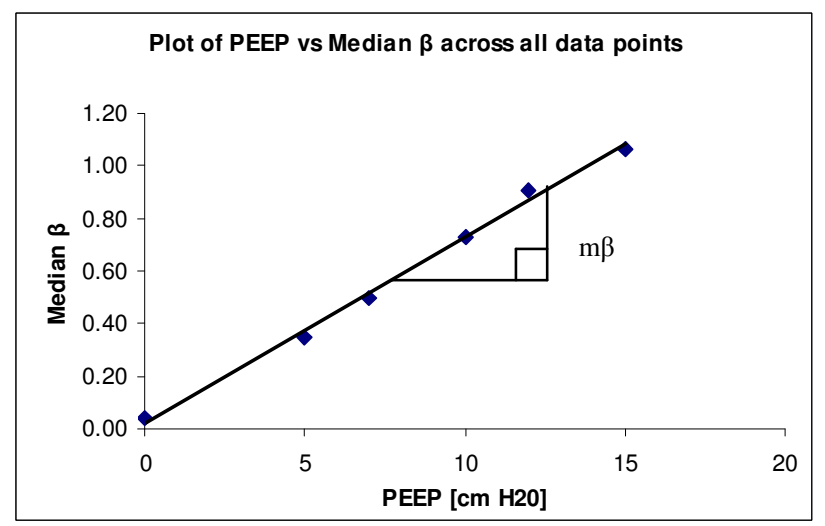

Figure 6 - Plot of Average Beta taken across all data points vs PEEP

Using these calculated parameters from $80 \%$ of the data, the EEV was then estimated for the remaining $20 \%$ of the data. A linear regression was performed by comparing the estimated EEV with the measured EEV for the remaining $20 \%$ of the data ( 8 data points). The process was then repeated 100,000 times and summary statistics reported.

This repetition ensures a wide and reasonably exhaustive coverage of possible test $(n=8)$ and validation $(n=32)$ sets are examined. This approach eliminates or reduces the 
potential that random chance or specific patient data sets skewed the results. It thus serves to statistically validate the general modelling and analysis approach presented.

Table 3 - 90\% Confidence Interval, Median and IQR of $R^{2}, m \beta$ and $\beta$ at all PEEP values

\begin{tabular}{|c|c|c|c|}
\hline & $90 \% \mathrm{CI}$ & Median & IQR \\
\hline $\mathbf{R}^{2}$ Coefficient & {$[0.910,0.960]$} & 0.944 & {$[0.933,0.952]$} \\
\hline $\mathbf{m} \boldsymbol{\beta}$ & {$[0.804,1.164]$} & 0.953 & {$[0.877,1.046]$} \\
\hline$\beta$ at $\mathrm{PEEP}=5 \mathrm{cmH}_{2} \mathrm{O}$ & {$[3.057,5.020]$} & 4.065 & {$[3.695,4.458]$} \\
\hline$\beta$ at $\mathrm{PEEP}=7 \mathrm{cmH}_{2} \mathrm{O}$ & {$[5.122,7.233]$} & 6.257 & {$[5.823,6.680]$} \\
\hline$\beta$ at $\mathrm{PEEP}=10 \mathrm{cmH}_{2} \mathrm{O}$ & $\begin{array}{l}{[7.883,} \\
10.190]\end{array}$ & 9.074 & {$[8.628,9.520]$} \\
\hline$\beta$ at $\mathrm{PEEP}=12 \mathrm{cmH}_{2} \mathrm{O}$ & $\begin{array}{l}10.235, \\
12.230]\end{array}$ & 11.371 & $\begin{array}{l}10.873, \\
11.887]\end{array}$ \\
\hline$\beta$ at $\mathrm{PEEP}=15 \mathrm{cmH}_{2} \mathrm{O}$ & $\begin{array}{l}{[12.430,} \\
15.170]\end{array}$ & 13.800 & $\begin{array}{l}{[12.512,} \\
15.170]\end{array}$ \\
\hline
\end{tabular}

Table 3 shows the statistical results for the $\mathrm{R}^{2}$ correlation coefficient between the estimated and actual EEV, $\mathrm{m} \beta$ and the $\beta$ at all PEEP levels. As shown, a 90\% confidence interval still produces a very high correlation coefficient. The low variability in $\mathrm{m} \beta$ and $\beta$ validates the assumption of population constants that can be used to describe any patient at this level of PEEP.

Figure (6) showed a linear relationship between $\beta$ and PEEP. At a PEEP $=0 \mathrm{cmH} 2 \mathrm{O}$, the dynamic FRC value should be zero, and hence the linear relationship between $\beta$ and PEEP is described as:

$$
\frac{\beta}{P E E P}=m \beta
$$

where the term $\beta /$ PEEP is equal to $m \beta$.

\section{DISCUSSION}

\subsection{Stress Strain Relationship}

The ability of using lung stress and strain as a proxy produces a reasonable estimate to the clinically measured EEV endpoint. The clinical data set used the study indicated that $\beta$ increased very linearly as a function of PEEP. Chiumello et al indicated that the ratio of lung elastance to total respiratory elastance $(\alpha)$ varied between a value of 0.33 to 0.95 over various PEEP settings. Gattitoni et al showed that the respiratory mechanics may vary in patients depending on whether the ARDS originated from pulmonary or extrapulmonary disease (Gattinoni '98). They concluded that depending on the origin of ARDS, the total respiratory elastance could increase or decrease as a function of PEEP increase. However, no significant changes for $\beta$ occured in this study, implying that $E_{\text {Lspec }}$ and $\alpha$ are interdependent on each other.

In this analysis, because $\beta$ incorporates the EEV, FRC, $E_{\text {Lspec }}$ and $\alpha$ according to Equation (9), as $\beta$ increases, EEV also increases linearly. Hence, $\beta$ is linearly related to EEV, so that as $\beta$ increases, EEV also increases in this model.

\subsection{Model Parameters}

The model uses 2 parameters to estimate the EEV for a given PEEP. The parameters are the $\beta$ and $\mathrm{m} \beta$, where $\mathrm{m} \beta$ is the gradient of the curve of $\beta$ vs PEEP as shown in Figure 6. Cross correlation and validation showed very tight ranges on a high $\mathrm{R}^{2}$ correlation coefficient. This result implies that $\beta$ and $\mathrm{m} \beta$ can be used as universal parameters across all PEEP levels to estimate the EEV and its change for a given PEEP, as well as for any ARDS patient. However, this conclusion must be subject to validation in a larger clinical trial given the small patient numbers available here.

\subsection{Clinical Implications}

The estimation of this EEV value could be very useful in the clinical setting. Clinicians can predict the amount of extra volume that can be recruited due to an increase in PEEP before applying it to a patient. To evaluate the EEV according to Equation (8), a series of inspiratory holds must be done to evaluate $\Delta \mathrm{V}_{\mathrm{EEV}} / \Delta \mathrm{P}_{\mathrm{PEEP}}$. Given three such results, it is possible to estimate the volume responsiveness of the patient and to apply Equation (8) to evaluate the $\mathrm{EEV}$. This is not an unreasonable intervention at bedside.

Another implication of this model is as a means to estimate FRC. According to Equation (9), $\beta$ is related to the value of $\alpha, E_{L s p e c}$ and EEV at a given PEEP. Using a median value of $\alpha=0.7$ and $E_{L s p e c}=13.5 \mathrm{~cm} \mathrm{H} 2 \mathrm{O}$, and based on the predicted EEV in this study, estimating FRC in Equation (9) gives an average value of FRC for ARDS patients as $875 \mathrm{mls}$. This value is within the values reported by Chiumello et al of FRC in ARDS patients of $1013 \pm 593 \mathrm{mls}$ and hence validates the model further.

In reality, the value of $\alpha$ varies and hence FRC can also take on a range of value. This range of FRC values can then give an indication of the level of recruited lung without any MV treatment and thus an indication of the total lung damage in the patient. It might thus also serve as an indication of when to begin weaning from MV.

\section{REFERENCES}

Amato, M. B. P., Barbas, C. S. V. et al. (1998). "Effect of a Protective-Ventilation Strategy on Mortality in the Acute Respiratory Distress Syndrome. " $\underline{\mathrm{N}}$ Engl J Med 338(6): 347-354.

Bersten, A. D. (1998). "Measurement of overinflation by multiple linear regression analysis in patients with acute lung injury." Eur Respir J 12(3): 526532.

Bersten, A. D., Edibam, C. et al. (2002). "Incidence and Mortality of Acute Lung Injury and the Acute Respiratory Distress Syndrome in Three 
Australian States." Am. J. Respir. Crit. Care Med. 165(4): 443-448.

Carlo, W. A. and Ambalavanan N. (1999). "Conventional Mechanical Ventilation: Traditional and New Strategies. "Pediatrics in Review 20(12): 117126.

Chiumello, D., Carlesso, E. et al. (2008). "Lung Stress and Strain during Mechanical Ventilation for Acute Respiratory Distress Syndrome." Am. J. Respir. Crit. Care Med. 178(4): 346-355.

Esteban, A., Anzueto, A. et al. (2002). "Characteristics and outcomes in adult patients receiving mechanical ventilation: a 28-day international study." Jama 287(3): 345-355.

Gattinoni, L., Pelosi, P. et al. (1998). "Acute Respiratory Distress Syndrome Caused by Pulmonary and Extrapulmonary Disease . Different Syndromes?" Am. J. Respir. Crit. Care Med. 158(1): 3-11.

Gattinoni, L. and Pesenti, A. (2005). "The concept of "baby lung"." Intensive Care Medicine 31(6): 776 - 784.

Halter, J. M., Steinberg, J. M. et al. (2003). "Positive EndExpiratory Pressure after a Recruitment Maneuver Prevents Both Alveolar Collapse and Recruitment/Derecruitment." Am. J. Respir. Crit. Care Med. 167(12): 1620-1626.

Heinze, H., Schaaf, B. et al. (2007). "The Accuracy of the Oxygen Washout Technique for Functional Residual Capacity Assessment During Spontaneous Breathing." Anesth Analg 104(3): 598-604.

Hughes, M., Grant, I. et al. (2000). "Incidence and Mortality after Acute Respiratory Failure and Acute Respiratory Distress Syndrome in Sweeden, Denmark and Iceland." Am. J. Respir. Crit. Care Med. 162(1): 332-333.

Malbouisson, L. M., Muller, J. C. et al. (2001). "Computed Tomography Assessment of Positive Endexpiratory Pressure-induced Alveolar Recruitment in Patients with Acute Respiratory Distress Syndrome." Am. J. Respir. Crit. Care Med. 163(6): 1444-1450.

McCann, U. G., Schiller, H. J. et al. (2001). "Visual validation of the mechanical stabilizing effects of positive end-expiratory pressure at the alveolar level." J Surg Res 99(2): 335-342.

Network., T. A. R. D. S. (2000). "Ventilation with lower tidal volumes as compared with traditional tidal volumes for acute lung injury and the acute respiratory distress syndrome." $\mathrm{N}$ Engl J Med 342(18): 1301-1308.

Walsh, T. S., Dodds, S. et al. (2004). "Evaluation of simple criteria to predict successful weaning from mechanical ventilation in intensive care patients." Br. J. Anaesth. 92(6): 793-799.

Ware, L. B. and Matthay M. A. (2000). "The Acute Respiratory Distress Syndrome." N Engl J Med 342(18): 1334-1349. 